\title{
Free Energy of the Hydrophobic Interaction from Molecular Dynamics Simulations: The Effects of Solute and Solvent Polarizability
}

\author{
Steven W. Rick \\ Frederick Biomedical Supercomputing Center, SAIC-Frederick, NCI-Frederick Cancer Research and \\ Development Center, Frederick, Maryland 27102
}

\author{
B. J. Berne* \\ Department of Chemistry and Center for Biomolecular Simulation, Columbia University, New York 10027
}

Received: May 12, 1997; In Final Form: September 2, $1997^{\circledR}$

\begin{abstract}
Molecular dynamics simulations are used to calculate the free energy of methane association in water, using the polarizable fluctuating charge model that treats the charges on atomic sites as dynamical variables. Compared with previous studies using nonpolarizable potentials, the inclusion of polarizability leads only to small differences in the methane pair potential of mean force. This is in contradistinction to two previous studies using other polarizable models, which do not agree with the nonpolarizable results or with each other. The potential of mean force is calculated at three different temperatures $(283,298$, and $313 \mathrm{~K})$ from which the temperature dependence and also the entropic part of the free energy is examined. It is found that the tendency for methane molecules to aggregate increases with increasing temperature and that aggregation is stablized by entropy.
\end{abstract}

\section{Introduction}

Nonpolar solutes have a strong tendency to aggregate in water due to the weakness of the solute-solvent interaction relative to the solvent-solvent interactions. ${ }^{1,2}$ The resulting hydrophobic interaction is important in many phenomena including the stability of proteins and membranes. ${ }^{3}$ Methane pairs provide a useful, simple model for the study of the hydrophobic interaction and have been the topic of many computational studies. ${ }^{4-17}$ Computational studies are especially important since these hydrophobic interactions are difficult to study experimentally because of the low solubility of nonpolar molecules. Through these studies several general features in the methane-methane potential of mean force have emerged. At $300 \mathrm{~K}$, the pair in direct contact (the contact pair) is more stable by about $0.5-$ $1.5 \mathrm{kcal} / \mathrm{mol}$ over distantly separated pairs (the isolated pair). The isolated pair is at a distance where the correlations between the two are small, although many simulations do not approach this limit. There is an additional minimum corresponding to the methane pairs being separated by a single solvation shell (the solvent-separated pair). However, these studies used different potentials and simulation methods, and some uncertainties remain in terms of the effects of polarization, temperature, and, to a lesser degree, pressure on the hydrophobic interaction.

The dipole moment of a water molecule in the liquid is enhanced over its gas-phase value of $1.85 \mathrm{D}$. Although the dipole moment of an individual molecule in the liquid cannot be directly measured, it has been estimated from dielectric measurements to be around 2.5 D. ${ }^{18}$ The water potentials used in the studies of the hydrophobic interaction treat this effect in different ways and can be grouped into three types: nonpolarizable (SPC, ${ }^{19} \mathrm{ST} 2,{ }^{20} \mathrm{ST} 4{ }^{15}$ ), which have partial charges that lead to dipole moments $(2.1-2.3 \mathrm{D})$ only slightly larger than the gas-phase value; polarizable (P-SWRK, ${ }^{18} \mathrm{PSPC}^{21}$ ), which have a permanent dipole moment of $1.85 \mathrm{D}$ and an inducible point dipole that leads to a total dipole moment of about $2.5 \mathrm{D}$,

\footnotetext{
${ }^{\otimes}$ Abstract published in Advance ACS Abstracts, October 15, 1997.
}

at $300 \mathrm{~K}$ and $1 \mathrm{~atm}$; extended nonpolarizable (SPC/E, $\left.{ }^{22} \mathrm{WK}^{23}\right)$, which have an enhanced permanent dipole moment around 2.5 $\mathrm{D}$ and an energy term that takes into account the polarization energy. Water molecules near nonpolar solutes will feel a different electric field than bulk water molecules, so that polarizability may influence the solvation of nonpolar solutes. However, general conclusions about the effects of the solvent polarizability are difficult to make. The two studies using extended nonpolarizable models give qualitatively different results. The study using the SPC/E potential by Dang finds results that are in close agreement with the SPC results, with a barrier height from the contact pair of $0.90 \mathrm{kcal} / \mathrm{mol} .{ }^{14}$ Using the WK potential, New and Berne find a much deeper contact pair minimum, of $1.5 \mathrm{kcal} / \mathrm{mol} .{ }^{16}$ Simulations based on polarizable models give quite different results. The study of van Belle and Wodak using the PSPC potential ${ }^{21}$ finds a deep contact pair minimum but only a very shallow solvent separated minimum. ${ }^{13}$ The study of New and Berne using the polarizable Sprik (PSRWK) model $^{18}$ finds a deep contact pair minimum and also a deep solvent-separated minimum, deeper than in the corresponding WK nonpolarizable model. ${ }^{16}$ This difference may be due to the strengths of the methane-water interaction and the treatment of periodic boundary effects, but the role of solvent polarizability remains unclear. Comparisons would be more meaningful if in models being compared the free energies of solvation of a single methane molecule were the same. This is not the case. All studies so far have treated methane as nonpolarizable, even though methane is more polarizable than water, ${ }^{24}$ and Backx and Goldman have postulated that solute polarizability should destabilize the contact pair.

Just as the solvation of a single nonpolar solute molecule has a large unfavorable entropy change, the hydrophobic interaction is believed to have a large entropic component. ${ }^{2}$ If there is a large entropic contribution, then the potential of mean force should show a strong temperature dependence. Entropic stablization of the contact pair would mean that the contact pair should become more stable as the temperature is increased. The integral equation theory study of Pratt and Chandler was the 
first to look at the temperature dependence of the hydrophobic interaction, for two hard spheres in water, using an experimental water-water pair correlation function. ${ }^{4}$ This study found that the contact pair did not become more stable at increasing temperatures. The results for continuous methane-water potentials, however, do show a temperature dependence consistent with entropic stablization. Smith, Zhang, and Haymet, using the SPC water potential and a united-atom Lennard-Jones potential for the methane molecules, find a difference in the free energy between the methane pair in contact and at a distance of $7 \AA$ of $-0.57 \pm 0.05 \mathrm{kcal} / \mathrm{mol}$ with the entropic contribution $(-T \Delta S)$ being $-1.5 \pm 0.8 \mathrm{kcal} / \mathrm{mol}$. This was for a constant $T, V$, and $N$ system. $^{11,12}$ Dang, using the SPC/E potential and an all-atom methane potential, reports the methane pair potential of mean force at two different temperatures (300 and $330 \mathrm{~K}$ ) also for a constant $T, V$, and $N$ ensemble. ${ }^{14}$ From the two curves, the entropic contribution at a temperature of $315 \mathrm{~K}$ can be calculated by finite difference. At this temperature, the free energy of the contact pair is about $-0.8 \mathrm{kcal} / \mathrm{mol}$ relative to the separated pair and the entropic contribution is about -1.6 $\mathrm{kcal} / \mathrm{mol}$. In other $N V T$ ensemble studies by Lüdemann et al., using SPC water and united-atom methane, the methane pair potential of mean force was calculated for temperatures ranging from 250 to $500 \mathrm{~K} .{ }^{17}$ Again, from finite difference, the entropy can be found. At $300 \mathrm{~K}$, the contact pair-separated pair free energy difference is $-0.5 \mathrm{kcal} / \mathrm{mol}$ and the entropic contribution is $-1.6 \mathrm{kcal} / \mathrm{mol}$. A study of four united-atom methane molecules in SPC water (in the $N, V, E$ ensemble) finds that the tendency of these methane molecules to aggregate increases with temperature. ${ }^{27}$

In addition to the constant volume calculations, the study by Dang also looked at the potential of mean force at the two different temperatures but using the correct densities of water at 300 and $330 \mathrm{~K}$ and $1 \mathrm{~atm} .{ }^{14}$ Under these conditions, the potential of mean force was the roughly the same at the two different temperatures, meaning that the entropy change is zero. The differences between the constant pressure and constant volume results of Dang suggest that there may be some important differences between the two ensembles. However, Lüdemann et al. also repeated their calculations adjusting for the density difference (at $350 \mathrm{~K}$ ) and found no difference between the potential of mean force at this temperature for the two different densities. ${ }^{17}$ This direct contradiction of the Dang results might be attributable to simulation details such as the box size and other sampling conditions. ${ }^{17}$

As all of these studies indicate, it is difficult to separate the effects of interest, such as polarization or temperature, from the other details of the potential that are not of principle interest, such as the methane-water potential or the treatment of periodic boundary conditions. In general, one would like to keep as much as possible in common between the different studies, but this can be difficult. For example, keeping the strength of the methane-water potential the same does not necessarily give the same solvation free energy for a single methane molecule for different water models, and this will effect the potential of mean force. To understand the influence of polarization, both of the solvent and of the solutes, and the temperature dependence on the hydrophobic interaction, we will use the fluctuating charge (FQ) model of water ${ }^{28}$ and a newly parametrized allatom FQ model for methane, described in the next section.

\section{Potential and Simulation Details}

The fluctuating charge (FQ) method for treating electronic polarization effects has been developed and applied to water ${ }^{28}$ and the solvation of amides. ${ }^{29}$ In one variation of the FQ model, the one used here, the charged sites in water are chosen to be the same as in the TIP4P model, ${ }^{30}$ and the partial charges on atomic sites are treated as variables that respond to changes in their environments. The FQ potential has Lennard-Jones and Coulombic interactions between different molecules and a molecular energy

$$
\begin{array}{r}
U(\{Q\},\{\boldsymbol{r}\})=\sum_{j<i} \sum_{\alpha} \sum_{\beta}\left(4 \epsilon_{\alpha \beta}\left[\left(\frac{\sigma_{\alpha \beta}}{r_{i \alpha, j \beta}}\right)^{12}--\left(\frac{\sigma_{\alpha \beta}}{r_{i \alpha, j \beta}}\right)^{6}\right]+\right. \\
\left.Q_{i \alpha} Q_{j \beta} / r_{i \alpha, j \beta}\right)+\sum_{i}\left(\sum_{\alpha} \tilde{\chi}_{\alpha}^{0} Q_{i \alpha}+{ }^{1} / 2 \sum_{\alpha} \sum_{\beta} Q_{i \alpha} Q_{i \beta} J_{\alpha \beta}\left(r_{i \alpha, i \beta}\right)-\right. \\
\left.E_{i}^{\mathrm{gp}}\right)(1)
\end{array}
$$

where $E_{i}^{\mathrm{gp}}$ is the gas-phase energy, $\tilde{\chi}_{\alpha}^{0}$ is the Mulliken electronegativity of the isolated atom, and $J_{\alpha \beta}\left(r_{i \alpha, i \beta}\right)$ is taken to be the Coulomb overlap integral between two Slater orbitals centered on each atomic site, which are characterized by a principal quantum number, $n_{\alpha}$, and an exponent $\zeta_{\alpha}$. The value of $J_{\alpha \alpha}(r)$ for $r=0$ is $J_{\alpha \alpha}^{0}$, and therefore the value of $\zeta_{\alpha}$ uniquely determines $J_{\alpha \alpha}^{0}$. For hydrogen, $n_{\mathrm{H}}=1$ and $J_{\mathrm{HH}}^{0}=$ $5 \zeta_{\mathrm{H}} / 8$, and for second-row elements, $n=2$ and $J_{\mathrm{AA}}^{0}=93 \zeta_{A} /$ 256. Notice that in the FQ model, unlike other molecular force fields, all charges on the same molecule interact, with the charges screened by $J_{\alpha \beta}(r)$. Beyond a distance of about $2.5 \AA$, $J_{\alpha \beta}(r)$ becomes equal to $1 / r$.

The set of charges which minimize eq 1 are the ground-state charges, subject to a charge neutrality constraint on each molecule. Since $\partial U / \partial Q$ is Mulliken's definition for electronegativity, the minimum energy charges will correspond to the electronegativity being equal at each site in a molecule. ${ }^{31-37}$ Rather than solving for the charges exactly at each time step, the method treats them as dynamical variables, which are propagated in an extended Lagrangian formalism at a low temperature so as to remain near the potential energy minimum. In the extended Lagrangian formalism, the charges are given a fictitious mass, chosen to be small enough so that the charges readjust rapidly to changes in the nuclear positions but also large enough so that a standard $1 \mathrm{fs}$ time step can be used. For the simulations reported here the charge masses are 0.6 for the water charges, 2.0 for the carbon atom charges, and 5.0 for the methyl hydrogens, all in units of $10^{-4}(\mathrm{ps} / e)^{2}$. In addition, no thermostat was used for the charges to keep them at a cold temperature, since there is little thermal coupling between the charge and position degrees-of-freedom. For the duration of a single 10 ps simulation, the charge temperature remains less than $5 \mathrm{~K}$.

The FQ electrostatic potential depends on the parameters $\tilde{\chi}_{\alpha}^{0}$ and $\zeta_{\alpha}$. By charge conservation, only the difference in $\tilde{\chi}$ values is important, so a molecule with $N$ different atom types is described by $N-1 \chi$ values. The Lennard-Jones term introduces two more parameters, which in the present calculation are placed only on the carbon and oxygen atoms. The parameters are chosen on the basis of a few known properties. First, like the earlier studies of amides, the electrostatic parameters are chosen to reproduce the gas-phase charges given by electronic structure calculations. ${ }^{29}$ Second, the model is designed to give accurate charges in the aqueous phase by choosing parameters so that the molecule when surrounded by a dielectric continuum has charges similar to those from electronic structure/dielectric continuum studies. Third, the Lennard-Jones parameters are chosen so that the minimum energy of the $\mathrm{CH}_{4}-\mathrm{H}_{2} \mathrm{O}$ dimer has a value and an oxygen carbon distance close to ab initio calculations at the fourthorder Møller-Plesset (MP4) level (Figure 1). ${ }^{38}$ Figure 1 also 


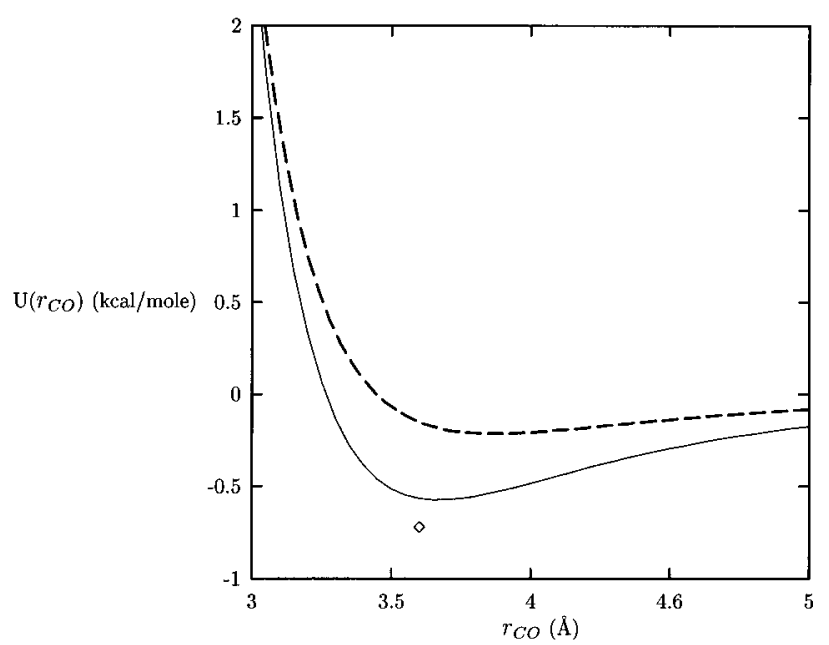

Figure 1. Fluctuating charge interaction energy between methane and water as a function of carbon-oxygen distance, with optimized hydrogen orientations (solid line), compared with the ab initio estimate (diamond) from ref 38 and the Jorgensen-Madura-Swenson SPC Lennard-Jones potential (dashed line) from refs 39 and 19.

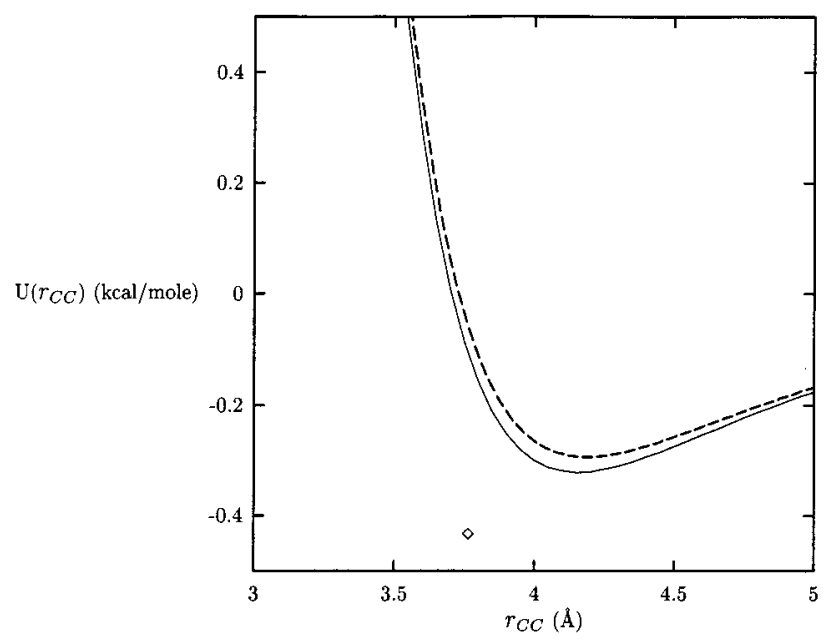

Figure 2. Fluctuating charge interaction energy between two methane molecules, with optimized hydrogen orientations (solid line), compared with the ab initio estimate (diamond) from ref 35 and the JorgensenMadura-Swenson Lennard-Jones potential (dashed line).

shows the Lennard-Jones interaction using the JorgensenMadura-Swenson TIP4P water-methane parameters $\left(\epsilon_{\mathrm{CO}}=\right.$ $\left.0.213 \mathrm{kcal} / \mathrm{mol}, \sigma_{\mathrm{CO}}=3.442 \AA\right) .{ }^{30,39}$ The Lennard-Jones parameters are also chosen so that the methane-methane interaction, when minimized with respect to the orientations of the hydrogens, is close to the Jorgensen-Madura-Swenson Lennard-Jones curve (Figure 2) since that has been shown to be an accurate potential. ${ }^{39}$ Notice that both the potentials are not nearly as deep as the MP2 estimate. ${ }^{35}$ One last property used is the solvation free energy, $\Delta G_{S}$. This last property provides the best estimate of the quality of the force field, since if the model is to give a good estimate for the thermodynamics of the methane pair, it should give good values for the single solute. Both potentials shown in Figure 1 give about the same solvation free energy, despite having a much different well depth, due to the fact that the water-water interactions are different. ${ }^{12,41,42}$ The parameters for methane, as well as the previously reported parameters for water, are given in Table 1. Lorentz-Berthelot combining rules for the Lennard-Jones parameters are used: the arithmetic mean for $\sigma_{i j}$ and geometric for $\epsilon_{i j}$.

The properties given by the FQ potential are shown in Table 2. The charges for gas-phase methane and small charge
TABLE 1: Potential Parameters for the Fluctuating Charge Model

\begin{tabular}{lcclcc}
\hline & & $\epsilon(\mathrm{kcal} / \mathrm{mol})$ & $\sigma(\AA)$ & $\tilde{\chi}(\mathrm{kcal} /(\mathrm{mol} e))$ & $\zeta\left(\AA^{-1}\right)$ \\
\hline \multirow{2}{*}{ water } & $\mathrm{H}$ & 0 & 0 & 0 & 1.70 \\
& $\mathrm{O}$ & 0.2862 & 3.159 & 68.49 & 3.08 \\
methane & $\mathrm{H}$ & 0 & 0 & 0 & 3.21 \\
& $\mathrm{O}$ & 0.2842 & 3.747 & 39.21 & 2.65
\end{tabular}

TABLE 2: Properties of the Fluctuating Charge (FQ) Model Compared with Electronic Structure and Experimental Results: The Gas-Phase Hydrogen Atom Charge $q_{\mathrm{H}}^{\mathrm{gp}}$, SelfConsistent Reaction Field Charge $q_{\mathrm{H}}^{\mathrm{aq}}$, Solvation Free Energy $\Delta G_{\mathrm{S}}$, Dipole Polarizability $\alpha$, Translational Diffusion Constant $D$, and Rotational Diffusion Constants for $\mathrm{CH}_{4}$ and $\mathrm{CD}_{4}, \tau_{2}$

\begin{tabular}{llcl}
\hline & \multicolumn{1}{c}{ FQ } & electronic structure & experiment \\
\hline$q_{\mathrm{H}}^{\mathrm{gp}}(e)$ & 0.125 & 0.125 & \\
$q_{\mathrm{H}}^{\mathrm{aq}}(e)$ & 0.129 & 0.130 & \\
$\alpha\left(\AA^{3}\right)$ & 1.09 & & $2.59^{a}$ \\
$\Delta G_{\mathrm{S}}(\mathrm{kcal} / \mathrm{mol})$ & $2.3 \pm 0.2$ & & $2.0^{b}$ \\
$D\left(\mathrm{CH}_{4}\right)\left(10^{-9} \mathrm{~m}^{2} / \mathrm{s}\right)$ & $1.8 \pm 0.3$ & & $2.4 \pm 0.5^{c}$ \\
$\tau_{2}\left(\mathrm{CH}_{4}\right)(\mathrm{ps})$ & $0.07 \pm 0.02$ & & \\
$\tau_{2}\left(\mathrm{CD}_{4}\right)(\mathrm{ps})$ & $0.11 \pm 0.04$ & & $0.12^{c}$
\end{tabular}

${ }^{a}$ Reference $24 .{ }^{b}$ Reference $26 .{ }^{c}$ Reference 45.

polarization induced by the reaction field are in good agreement with the electrostatic potential (ESP) fitted charges from electronic structure and self-consistent reaction field (SCRF) calculations, which were done using the PSGVB program with a 6-31G** basis set. ${ }^{43}$ Both the SCRF and the FQ calculations were done with a carbon atomic radius of $1.9 \AA$ and a hydrogen atomic radius of $1.15 \AA$. The $\mathrm{FQ} /$ continuum calculations were done using the DelPhi ${ }^{44}$ program in a self-consistent manner as described previously. ${ }^{29}$

The FQ dipole polarizability is $1.1 \AA{ }^{3}{ }^{3}$ less than the experimental value of $2.6 \AA^{3},{ }^{24}$ so the FQ model and perhaps the HF calculation on which it is based underestimate the dipole polarizability (although the HF calculations should not be that far off). On the other hand, some of the dipole polarizability is not due to charge transfer between atoms, which is what the FQ model treats, but rather due to local polarization of the charge distribution around the central carbon atom, which gives rise to the dispersion term in the Lennard-Jones interaction. For this reason, the parameters were fit to values of the induced charges by the reaction field, rather than the experimental dipole polarizability, and some underestimation of the dipole polarizability is expected, although the FQ parameters used here may be underestimating it by too much.

While the current study is concerned with thermodynamics, dynamical properties provide another test of the accuracy of the potential. Measurements of the translational diffusion constant of methane in water show that it is about the same as that of bulk water. ${ }^{45}$ This is what this potential model gives, since the diffusion constant of TIP4P-FQ water is $(1.9 \pm 0.1)$ $\times 10^{-9} \mathrm{~m}^{2} / \mathrm{s}^{28}$ The rotational time scale is calculated from the time autocorrelation function of $P_{2}[\mathbf{e}(t) \cdot \mathbf{e}(0)]$, where $\mathbf{e}$ is a unit vector that points along the $\mathrm{CH}$ bond. ${ }^{45,46}$ The rotational time scale is fast, in agreement with the nuclear magnetic resonance (NMR) results. ${ }^{45}$

The simulations performed for the dynamical properties were done in the microcanonical (constant $E, V, N$ ) ensemble. All of the other simulations were done in the isothermal-isobaric (constant $T, P, N$ ) ensemble, by coupling to a pressure bath and a Nosé-Hoover temperature bath. ${ }^{47-51}$ The simulations are done at a temperature of $298 \mathrm{~K}$ and, to study the temperature dependence and therefore the entropy, at temperatures 283 and $303 \mathrm{~K}$, all at a pressure of $1 \mathrm{~atm}$. All simulations used a $1 \mathrm{fs}$ 


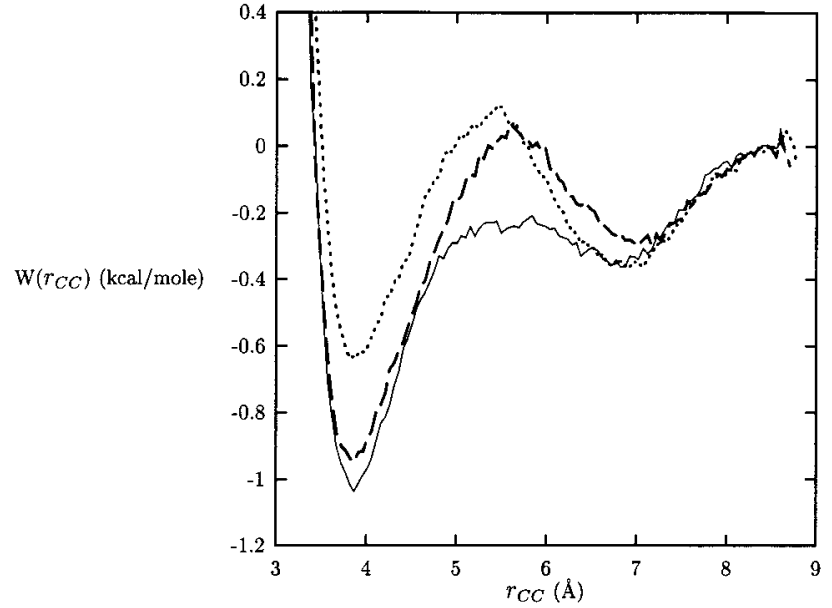

Figure 3. Methane pair potential of mean force at $T=283 \mathrm{~K}$ (dotted line), $298 \mathrm{~K}$ (solid line) and $313 \mathrm{~K}$ (dashed line).

time step, 256 solvent molecules, and the Ewald sum for longranged electrostatic interactions, and bond constraints were enforced using the SHAKE algorithm. ${ }^{52}$ The solvation free energy was calculated from about 2 ns of simulation data using using the same thermodynamic integration procedure as described elsewhere. ${ }^{29}$ The potential of mean force calculations were done using umbrella sampling, with a quartic restraining potential, $1 /{ }_{2} K\left(r_{\mathrm{CC}}-r_{0}\right)^{4}$ on the methane carbon distance, $r_{\mathrm{CC}}$, with five windows centered an angstrom apart from $r_{0}=4-8$ $\AA$ with a force constant, $K$, equal to $40 \mathrm{kcal} / \mathrm{mol} / \AA^{4}$. Each window was simulated for $1.2 \mathrm{~ns}$, and at the lower temperature, $283 \mathrm{~K}$, each window was simulated for $1.5 \mathrm{~ns}$. The potential of mean force was calculated from the biased data using the weighted histogram method. ${ }^{53,54}$

\section{Results}

The methane pair potential of mean force at three different temperatures is shown in Figure 3. At $298 \mathrm{~K}$, the contact pair minimum is $1.0 \mathrm{kcal} / \mathrm{mol}$ and that of the solvent-separated pair is $0.3 \mathrm{kcal} / \mathrm{mol}$, both relative to the isolated pair. The error estimates for these calculations representing a standard deviation are $0.2 \mathrm{kcal} / \mathrm{mol}$. This is about the same as the nonpolarizable SPC and SPC/E results. ${ }^{12-17}$ The most significant difference is the barrier between the contact pair and the solvent-separated pair, which is smaller for the FQ $(0.8 \mathrm{kcal} / \mathrm{mol})$ than for the SPC and SPC/E results $(1.0 \mathrm{kcal} / \mathrm{mol})$. For those studies the barrier represents the global maximum of $w(r)$, whereas for the FQ $w(r)$ the barrier free energy is below that of the isolated pair. The FQ result is in disagreement with the two other studies using polarizable water, most significantly in the region of the solvent-separated pair, which is deeper than the results of van Belle and Wodak ${ }^{13}$ and not as deep as the results of New and Berne. ${ }^{16}$ The stabilities of the contact pair and solvent-separated pair can be quantified by defining an equilibrium constant where $[\mathrm{CP}]$ and $[\mathrm{SSP}]$ are

$$
K_{\mathrm{eq}}=[\mathrm{SSP}] /[\mathrm{CP}]=\int_{r_{1}}^{r_{2}} 4 \pi r^{2} \mathrm{e}^{-w(r) / k T} / \int_{0}^{r_{2}} 4 \pi r^{2} \mathrm{e}^{-w(r) / k T} \mathrm{~d} r
$$

the concentrations of the contact pair and solvent-separated pair, respectively, and $r_{1}$ and $r_{2}$ define the limits of the contact and solvent-separated pair regions. ${ }^{5}$ Using $r_{1}=5.5 \AA$ and $r_{2}=8.5$ $\AA$, our present calculations give $K_{\text {eq }}=1.9,1.9,3.1$ respectively for temperatures $T=313 \mathrm{~K}, 298 \mathrm{~K}, 283 \mathrm{~K}$, meaning that the solvent-separated pair is more probable than the contact pair, at least partially because the solvent-separated region occupies a larger volume. Most of the reported $K_{\text {eq }}$ values in the literature

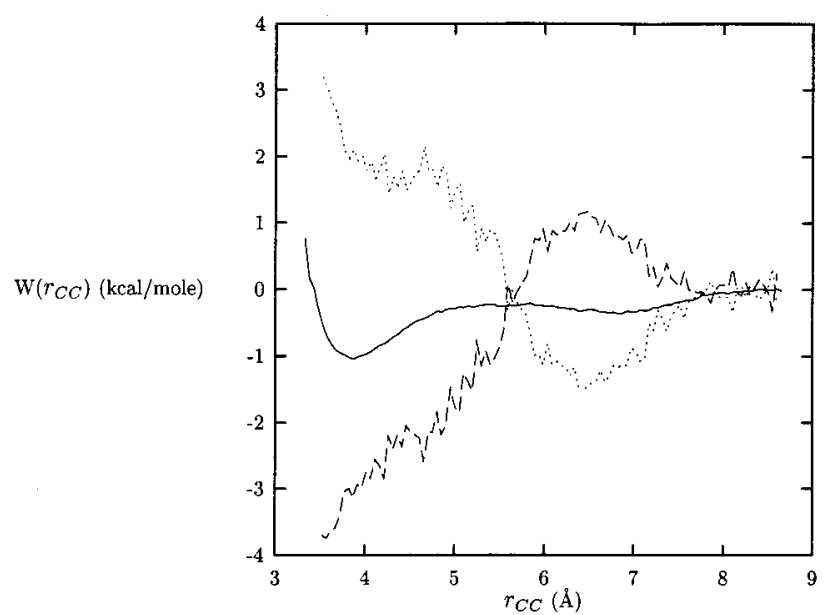

Figure 4. Methane pair potential of mean force at $T=298 \mathrm{~K}$ (solid line), together with the entropic, $-T \Delta S(r)$ (dashed line), and enthalpic contributions (dotted line).

are greater than 1, although the value of $K_{\text {eq }}$ is strongly dependent on the choice of $r_{2}{ }^{5,16,17}$ The exception is the $w(r)$ using the WK water model, which finds $K_{\text {eq }}$ equal to 0.7 , and so the contact pair is more probable. ${ }^{16}$

In our work, the contact pair become less stable at our lowest temperature of $283 \mathrm{~K}\left(K_{\mathrm{eq}}=3.1\right)$ while at the higher temperatures of 298 and $313 \mathrm{~K}, K_{\mathrm{eq}}=1.9$. A significant difference at higher temperatures is that the barrier between the $\mathrm{CP}$ and the SSP gets larger. While the contact pair does not get more stable at the higher temperature, the temperature differences are relatively small $(15 \mathrm{~K})$, and larger temperature increases might be necessary to determine if the CP becomes more stable at $T$ $>298 \mathrm{~K}$. The temperature difference was chosen to be small enough so that approximating $\Delta S(r)$ by $-(w(r ; T+\Delta T)-$ $-w(r ; T-\Delta T) /(2 \Delta T)$ is a good approximation. ${ }^{12}$ Lüdemann et al. use temperature differences of $50 \mathrm{~K}$ and find a more stable $\mathrm{CP}$ at $350 \mathrm{~K}$ than at $300 \mathrm{~K} .{ }^{17}$ In the temperature range $283-$ $313 \mathrm{~K}$, we do see a more stable CP. The entropy along with $w(r)$ and the enthalpic contribution to the free energy $(w(r)+$ $T \Delta S(r)$ ) are shown in Figure 4. From this figure, it is clear that there is a large entropic stablization of the $\mathrm{CP}$, by about 3 $\mathrm{kcal} / \mathrm{mol}$. In addition, the SSP and part of the barrier region are entropically unfavorable relative to the isolated pair by about $1 \mathrm{kcal} / \mathrm{mol}$. So the SSP is more stable at lower temperatures. Smith and Haymet, with the SPC potential, find that the CP is stablized by $1.6 \mathrm{kcal} / \mathrm{mol}$ and find a small entropy increase in the barrier region around $6 \AA .^{12}$ In the simulations of Lüdemann et al., the SSP minimum at the lowest temperature $(250 \mathrm{~K})$ appears to be as deep or deeper than at higher temperatures, although the differences are small. ${ }^{17}$

Next, we address the question of the methane's polarizability: does it tend to stablize the CP or the SSP? Over the range of methane separations and temperature, the charges of the methane molecule remain constant $\left(\mathrm{q}_{\mathrm{H}}=0.126 e\right)$. Since the potential is not pairwise additive, we cannot define a total methane-methane interaction, but we can look at individual contributions to the energy. The electrostatic interaction between the two methane molecules is very weak (about -0.005 $\mathrm{kcal} / \mathrm{mol}$ at the $\mathrm{CP}$ distance), implying that methane's polarizability, as treated here, does not seem to change the relative stabilities of the CP and the SSP. For more polarizable solutes, such as xenon, solute polarizability may change the stability of the CP. ${ }^{25}$

These calculations are done at constant pressure, whereas most of the previous calculations of the methane pair potential of mean force were done at constant volume. As stated in the 
Introduction, there has been some discussion in the literature about differences between constant pressure versus constant volume, particularly how it relates to the temperature dependence. ${ }^{27,14,17}$ Here we ask a different question: does the volume change as a function of $r_{\mathrm{CC}}$, therefore changing the potential of mean force relative to constant volume calculations? The answer is no. The volume is essentially constant as a function of $r_{\mathrm{CC}}$, meaning there is no significant $P \Delta V$ contribution to the free energy.

\section{Conclusions}

Using the polarizable FQ method for both the solute and the solvent, we find that the methane pair potential of mean force, at $1 \mathrm{~atm}$ and $298 \mathrm{~K}$, has two minima, with the contact pair being more stable by about $0.7 \mathrm{kcal} / \mathrm{mol}$. This is in qualitative agreement with the results using nonpolarizable potentials ${ }^{12-17}$ and not in agreement with other studies using polarizable water models. ${ }^{13,16}$ The polarizable studies disagree with each other as well; the SSP minimum is very shallow in the van Belle and Wodak PSPC study ${ }^{13}$ and very deep in the New and Berne P-SWRK study. ${ }^{16}$ The strength of the methane-water interactions may explain these differences, rather than the water-water interactions. For the polarizable models of refs 13 and 16, the solvation free energy has not been reported. In the present study, the solvation free energy is in good agreement with the nonpolarizable SPC water and Jorgensen-Madura-Swenson methane model (and in good agreement with experiment), despite the fact that the methane-water interactions are very different (Figure 1). This may explain the agreement in the methane pair results.

The Watanabe-Klein water-water nonpolarizable potential has a strength comparable to the FQ potential, with LennardJones parameters ( $\sigma=3.18 \AA, \epsilon=0.281 \mathrm{kcal} / \mathrm{mol})$ like those of the FQ model (see Table 1) and fixed charges that are the same as the average value for the aqueous $\mathrm{FQ}$ charges $\left(\mathrm{q}_{\mathrm{H}}=\right.$ $0.6 e) .{ }^{23}$ New and Berne ${ }^{16}$ calculated the methane pair potential using this potential and united-atom, purely Lennard-Jones water-methane interaction with $\sigma=3.44 \AA$ and $\epsilon=0.213$ $\mathrm{kcal} / \mathrm{mol}$ similar to the Jorgesen-Madura-Swenson SPC interaction shown in Figure 1. ${ }^{19,39}$ Those calculations find a contact pair that is very stable relative to the solvent-separated pair, by more than $1 \mathrm{kcal} / \mathrm{mol}$, whereas the FQ model finds the two minima are separated by only $0.7 \mathrm{kcal} / \mathrm{mol}$. Some of these differences might be due to polarizability, but they can be partially rationalized in terms of the differences in the methanewater interaction. For the contact pair, each methane molecule will have one less water and one more methane nearest-neighbor relative to the solvent-separated pair. Neglecting the electrostatic parts, the contribution of the methane-water interaction to the free energy difference between the solvent-separated and contact pair is $2 \epsilon_{\mathrm{WM}}-\epsilon_{\mathrm{MM}}$, where $\epsilon_{\mathrm{WM}}$ is the water-methane well depth and $\epsilon_{\mathrm{MM}}$ the methane-methane well depth. ${ }^{4}$ This value is $0.15 \mathrm{kcal} / \mathrm{mol}$ larger for the parameters used in the New and Berne study, so their deeper contact pair minimum is consistent with this analysis.

The polarizability of the solute does not influence the potential of mean force. The methane charges turn out to be constant as a function of methane pair distance and are small $\left(q_{\mathrm{H}}=0.126 \mathrm{e}\right)$, so that the electrostatic interactions are weak, weaker than the Lennard-Jones interactions. Solute polarizability is an important factor in other aqueous systems, such as conformational equilibria of amides ${ }^{29}$ and chloride ion water clusters. ${ }^{56}$ It thus seems that nonpolarizable potentials are adequate to describe the hydrophobic interaction.

The potential of mean force has a temperature dependence with the contact pair becoming less stable at the lower temperature (Figure 3), in agreement with earlier studies ${ }^{17,27}$ (but see ref 14). From results at different temperatures, the entropy can be calculated and, at $298 \mathrm{~K}$, a large entropic stablization of the contact pair is found (Figure 4), which has also been reported in an earlier study. ${ }^{11,12}$ The solvent-separated pair and part of the barrier region are entropically destabilized. Additionally, the present calculations are done at constant pressure, rather than the previous calculations that were done at constant volume. However, we find a volume that is constant as a function of the methane pair distance, indicating that constant volume and constant pressure calculations should give similar results, as long as the correct volume is used.

Acknowledgment. We would like to thank Bryan Marten for performing the PSGVB calculations. Part of this research was funded by a grant from NIH.

\section{References and Notes}

(1) Franks, F. Water-A Comprehensive Treatise; Franks, F., Ed.; Plenum: New York, 1972; Vol. 1.

(2) Ben-Naim, A. Hydrophobic Interactions; Plenum: New York, 1980.

(3) Tanford, C. The Hydrophobic Effect: Formation of Micelles and Biological Membranes; Wiley: New York, 1980.

(4) Pratt, L. R.; Chandler, D. J. Chem. Phys. 1977, 67, 3683.

(5) Pangali, C.; Rao, M.; Berne, B. J. J. Chem. Phys. 1979, 71, 2975.

(6) Swaminathan, S.; Beveridge, D. L. J. Am. Chem. Soc. 1979, 101, 5832 .

(7) Ravishanker, G.; Mezei, M.; Beveridge, D. L. Faraday Symp. Chem Soc. 1982, 17, 79.

(8) Rapaport, D. C.; Scheraga, H. A. J. Phys. Chem. 1982, 86, 873.

(9) Watanabe, K.; Andersen, H. C. J. Phys. Chem. 1986, 90, 795.

(10) Jorgensen, W. L.; Buckner, J. K.; Boudon, S.; Tirado-Rives, J. J. Chem. Phys. 1988, 89, 3742.

(11) Smith, D. E.; Zhang, L.; Haymet, A. D. J. J. Am. Chem. Soc. 1992, 114,5875 .

(12) Smith, D. E.; Haymet, A. D. J. J. Chem. Phys. 1993, 98, 6445.

(13) van Belle, D.; Wodak, S. J. J. Am. Chem. Soc. 1993, 115, 647.

(14) Dang, L. X. J. Chem. Phys. 1994, 100, 9032.

(15) Head-Gordon, T. Chem. Phys. Lett. 1994, 227, 215

(16) New, M. H.; Berne, B. J. J. Am. Chem. Soc. 1995, 117, 7172.

(17) Lüdemann, S.; Schreiber, H.; Abseher, R.; Steinhauser, O. J. Chem. Phys. 1996, 104, 286.

(18) Sprik, M. J. Chem. Phys. 1991, 95, 6762.

(19) Berendsen, H. J. C.; Postma, J. P. M.; von Gunsteren, W. F.; Hermans, J. Intermolecular Forces; Pullman, B., Ed.; Reidel: Dordrecht, Holland, 1981; p 331.

(20) Stillinger, F. H.; Rahman, A. J. Chem. Phys. 1974, 60, 1545.

(21) Ahlström, P.; Wallqvist, A.; Engström, S.; Jönsson, B. Mol. Phys. 1989, 68, 563.

(22) Berendsen, H. J. C.; Grigera, J. R.; Straatsma, T. P. J. Phys. Chem. 1987, $91,6269$.

(23) Watanabe, K.; Klein, M. L. Chem. Phys. 1989, 131, 157.

(24) Weast, Robert C., Ed. CRC Handbook of Chemistry and Physics, 66th ed.; CRC Press: Boca Raton, Fl, 1985-1986.

(25) Backx, P.; Goldman, S. Chem. Phys. Lett. 1985, 113, 578.

(26) Ben-Naim, A.; Marcus, Y. J. Chem. Phys. 1984, 81, 2016.

(27) Skipper, N. Chem. Phys. Lett. 1993, 207, 424.

(28) Rick, S. W.; Stuart, S. J.; Berne, B. J. J. Chem. Phys. 1994, 101, 6141.

(29) Rick, S. W.; Berne, B. J. J. Am. Chem. Soc. 1996, 118, 672.

(30) Jorgensen, W. L.; Chandrasekhar, J.; Madura, J. D.; Impey, R. W.;

Klein, M. L. J. Chem. Phys. 1983, 79, 926.

(31) Sanderson, R. T. Science 1951, 114, 670.

(32) Parr, R. G.; Yang, W. Density-Functional Theory of Atoms and Molecules; Oxford University Press: Oxford, 1989.

(33) Došen-Mićović, L.; Jeremić, D.; Allinger, N. L. J. Am. Chem. Soc. 1983, 105, 1716.

(34) Mortier, W. J.; Van Genechten, K.; Gasteiger, J. J. Am. Chem. Soc. 1985, 107,829 .

(35) No, K. T.; Grant, J. A.; Scheraga, H. A. J. Phys. Chem. 1990, 94, 4732 .

(36) Rappéand, A. K.; Goddard, W. A. J. Phys. Chem. 1991, 95, 3358.

(37) Reynolds, C. A.; Essex, J. W.; Richards, W. G. J. Am. Chem. Soc. 1993, $114,9075$.

(38) Szczęśniak, M. M.; Chahsiński, G.; Cybulski, S. M.; Cieplak, P. J. Chem. Phys. 1993, 98, 3078.

(39) Jorgensen, W. L.; Madura, J. D.; Swenson, C. J. J. Am. Chem. Soc. 1984, 106, 6638. 
(40) Novoa, J. J.; Whangbo, M. H.; Williams, J. M. J. Chem. Phys. 1991, 94, 4835.

(41) Jorgensen, W. L.; Blake, J. F.; Buckner, J. K. Chem. Phys. 1989, 129, 193.

(42) Guillot, B.; Guissani, Y.; Bratos, S. J. Chem. Phys. 1991, 95, 3643.

(43) Friesner, R. A. Annu Rev. Phys. Chem. 1991, 42, 341. Ringnalda, M.; Langlois, J. M.; Greeley, B.; Murphy, R.; Russo, T.; Cortis, C.; Muller, R.; Marten, B.; Donnelly, R., Jr.; Mainz, D.; Wright, J.; Pollard, W. T.; Cao, Y.; Won, Y.; Miller, G.; Goddard, W. A., III; Friesner, R. A. PSGVB; Schrodinger, Inc., 1994.

(44) Nicholls, A.; Honig, B. J. Comput. Chem. 1991, 12, 435.

(45) Laaksonen, A.; Stilbs, P. Mol. Phys. 1991, 74, 747.

(46) Impey, R. W.; Madden, P. A.; McDonald, I. R. Mol. Phys. 1982, $46,513$.
(47) Andersen, H. C. J. Chem. Phys. 1980, 72, 2384.

(48) Ciccotti, G.; Ryckaert, J. P. Comput. Phys. Rep. 1986, 4, 345.

(49) Martyna, G. J.; Tobias, D. J.; Klein, M. L. J. Chem. Phys. 1994 101, 4177.

(50) Nosé, S. Mol. Phys. 1984, 52, 255.

(51) Hoover, W. G. Phys. Rev. A 1985, 31, 1695.

(52) Allen, M. P.; Tildesley, D. J. Computer Simulation of Liquids; Oxford University Press: Oxford, 1987.

(53) Ferrenberg, A. M.; Swendsen, R. H. Phys. Rev. Lett. 1989, 63, 1195.

(54) Kumar, S.; Bouzida, D.; Swendsen, R. H.; Kollman, P. A.; Rosenberg, J. M. J. Comput. Chem. 1992, 13, 1011.

(55) Pratt, L. R.; Chandler, D. J. Chem. Phys. 1980, 73, 3434

(56) Stuart, S. J.; Berne, B. J. J. Phys. Chem. 1996, 100, 11934. 